\title{
REAL-TIME EXPERIMENTAL DEMONSTRATION OF PRECISE DECENTRALIZED RELATIVE NAVIGATION FOR FORMATION FLYING SPACECRAFT
}

\author{
Franz D. Busse* and Jonathan P. How ${ }^{\dagger}$
}

\begin{abstract}
This paper presents the first set of hardware-in-theloop results that experimentally demonstrate precise relative navigation for true formation-flying spacecraft applications. Carrier Differential-phase GPS provides an ideal navigation sensor for formation flying missions because it can be used to directly measure the relative positions and velocities of the vehicles in the fleet. A decentralized algorithm for formation estimation is presented, using an Adaptive Extended Kalman Filter to achieve unprecedented levels of accuracy. Four modified GPS receivers were used in the NASA Goddard Formation Flying Testbed facility to demonstrate formation flying in low Earth orbit. Results from these hardware-in-theloop tests show accuracies on the order of $\sim 1 \mathrm{~cm}$ position and $\sim 0.5 \mathrm{~mm} / \mathrm{s}$ for a $1 \mathrm{~km}$ elliptical formation. These results validate both the use of a decentralized architecture and the adaptive EKF.
\end{abstract}

\section{INTRODUCTION}

There has been significant recent interest from NASA and DoD in using formation flying spacecraft for a variety of earth and space science missions. For the purposes of this discussion, a formation is a group of vehicles where the relative state precision between vehicles in the group is of greater importance than the absolute state precision of those individual vehicles. Many potential benefits are foreseen through formation flying: it can bring down total mission cost, speed mission time-lines, add flexibility and

\footnotetext{
* Stanford University, fbusse@stanfordalumni.org

$\dagger$ Senior Member AIAA, Massachusetts Institute of Technology, jhow@mit.edu

Copyright (C) 2002 by the American Institute of Aeronautics and Astronautics, Inc. All rights reserved.
}

modularity to missions, and in some cases, it is required to meet the mission objective. ${ }^{1,2}$

Formation flying requires precise relative navigation. For some formation missions, such as interferometry, the relative navigation performance directly impacts the success of the primary mission. All formation missions, however, are aided by precise relative navigation. Tillerson demonstrates that fuel usage for active control of formation flying satellites is a strong function of relative navigation error, and in particular the relative velocities. ${ }^{3,4}$ The larger the error, the higher the fuel usage will be, and the shorter the mission life. So it is desirable for all formation flying satellite missions to achieve precise relative navigation.

To perform relative navigation, Carrier-Phase Differential GPS (CDGPS) provides an ideal sensor. It provides absolute and relative navigation, with centimeter level precision. GPS receivers have already been demonstrated for absolute navigation and also code phase relative navigation in the orbital environment. For example, researchers at NASDA used differential GPS during the ETS-VII mission, which involved two satellites performing a rendezvous. ${ }^{5}$ Relative position estimate accuracies were better than $10 \mathrm{~m}$ and relative velocity accuracy better than 3 $\mathrm{cm} / \mathrm{s}$ during maneuvers. Carpenter contains a good summary of recent orbital experiment results. ${ }^{6}$ As for simulation results, recent work at the University of Texas developed a closed-loop hardware simulation for rendezvous $(6 \mathrm{~cm}$ position error and $1 \mathrm{~mm} / \mathrm{s}$ velocity error) using CDGPS. ${ }^{7}$

This work uses a custom modified CDGPS receiver to perform relative (and absolute) navigation for a Low Earth Orbit satellite formation of four vehicles. A decentralized real-time estimation archi- 


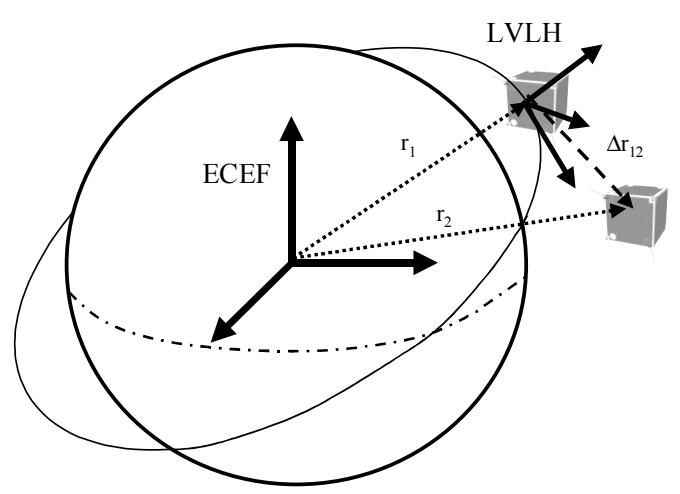

Fig. 1: Shows the absolute and relative states of a formation. Absolute states are expressed in the $E C E F$ frame. The Local-Vertical Local-Horizontal frame often provides an intuitive framework for the relative states.

tecture is implemented. To perform relative state estimation, an adaptive extended Kalman filter is implemented that requires very limited model information to process the measurements. Results are presented from actual hardware-in-the-loop experiments performed at the NASA Goddard Space Flight Center Formation-Flying Testbed facility. The results presented are, to the best knowledge of the authors, the best orbital relative navigation results that have ever been reported, and substantiates earlier expectations derived from software simulations. ${ }^{8,9}$ This work also experimentally validates the use of a decentralized estimation architecture.

\section{CENTRALIZED VS. DECENTRALIZED}

There are various strategies for estimating the formation state. ${ }^{10}$ The two archetypal approaches are centralized and decentralized. In a centralized approach, the measurements are sent from each slave to a single processor. There, all measurements are combined into a single filter, and the formation state is estimated. However, for formation flying, this centralized approach is less desirable. It requires the update and inverse of very large state covariance and measurement matrices. This results in a significant computational load, which is carried only by single a processor. Furthermore, this approach is not robust to a single-point failure, and it is not reconfigurable or flexible. And if the formations increase in size, then this centralized computation could become extremely difficult to perform in real-time.

In a decentralized approach, each slave vehicle performs state estimation, and the solutions are then collected by the master to form an entire fleet estimate, if necessary. Here, the computational load is spread across the vehicles in the formation. Note that for estimation, the difference between a master and slave node is only in software, and can be reconfigured during the mission at will. If the master fails, any other slave can easily replace it because the master is not special in any particular way. This makes the decentralized formation more robust. The formation can also be scaled to any number of vehicles. There is no cost difference between master and slave nodes. Logistically, the decentralized approach is more appealing. ${ }^{11}$ The critical question, however, is how do the two approaches impact the estimation accuracy?

Consider the formation state, $\mathcal{X}$, which is defined as $\mathcal{X}=\left[\begin{array}{lll}\mathbf{X}_{1}^{T} & \ldots & \mathbf{X}_{N}^{T}\end{array}\right]^{T} . \mathbf{X}_{i}$ is the state vector of the $i^{t h}$ vehicle in the formation. This formation state can also be expressed as

$$
\begin{aligned}
\mathcal{X}=\left[\begin{array}{c}
\mathbf{X}_{1} \\
\mathbf{X}_{2} \\
\vdots \\
\mathbf{X}_{N}
\end{array}\right]= & {\left[\begin{array}{c}
\mathbf{X}_{1} \\
\mathbf{X}_{1}+\Delta \mathbf{X}_{2-1} \\
\vdots \\
\mathbf{X}_{1}+\Delta \mathbf{X}_{N-1}
\end{array}\right] } \\
= & {\left[\begin{array}{c}
\mathbf{X}_{1} \\
\mathbf{X}_{1} \\
\vdots \\
\mathbf{X}_{1}
\end{array}\right]+\left[\begin{array}{c}
0 \\
\Delta \mathcal{X}
\end{array}\right] }
\end{aligned}
$$

where $\Delta \mathcal{X}$ is defined as

$$
\Delta \mathcal{X}=\left[\begin{array}{c}
\Delta \mathbf{X}_{2-1} \\
\vdots \\
\Delta \mathbf{X}_{N-1}
\end{array}\right]
$$

The formation state is completely described by either $\mathcal{X}$ or by $\left[\begin{array}{ll}\mathbf{X}_{1}^{T} & \Delta \mathcal{X}^{T}\end{array}\right]^{T}$. Note that in this derivation, the subscript 1 indicates the master vehicle, and all others would be considered slave vehicles. For state estimation, the selection of the master is arbitrary (it could be any vehicle in the formation).

To perform relative navigation using GPS, a GPS receiver is placed on each vehicle, so there exists for each vehicle a set of measurements, $\mathbf{Y}_{i}=h\left(\mathbf{X}_{i}, E_{i}\right)$. 
These measurements are a function of two fundamental quantities: the vehicle state, $\mathbf{X}$, and the environment of the vehicle, $E$. For our GPS application, the environment includes the NAVSTAR satellite states and ionosphere. The measurements from each vehicle are independent of the state of any other vehicle. However, since the vehicles in the formation are usually in close proximity to each other, the environments across the formation are often similar $\left(E_{1} \approx E_{2} \approx \cdots \approx E_{N}\right)$, and so the measurement sets are correlated.

If each slave estimates its own state $\left(\hat{\mathbf{X}}_{i}\right)$, and then returns that estimate to the master to create a formation state estimate, the differential state error will be on the order of two times the absolute state error. This has already been experimentally demonstrated many times (a thorough example was performed by Binning in Ref. [12]).

However, if the "master" vehicle broadcasts its measurements $\left(\mathbf{Y}_{1}\right)$ out to the slave vehicles, then they can determine their state relative to the master, $\Delta \hat{\mathbf{X}}_{i-1}$ using the differential measurements $\Delta \mathbf{Y}_{i-1}{ }^{*}$. These differential measurements have much smaller errors associated with them (since the common terms are canceled out). The differential unmodeled process noises are also much smaller than the absolute process noises. These factors make the relative state estimate using differential measurements much more accurate than just differencing the absolute solutions. The differential approach has the additional advantage of directly estimating $\Delta \hat{\mathcal{X}}$, which for a formation is of greater interest than $\hat{\mathcal{X}}$. For a decentralized algorithm, it is best to compute the differential states.

To experimentally validate this, more than two vehicles are required. Two vehicles provide only one differential measurement set and differential state estimate; three or more vehicles are required then to compare differential state estimates. All previous orbital relative navigation work has used only two vehicles, so the degree of correlation between differential state estimates could never be observed.

For logistical reasons of robustness, computational load distribution, and scalability, we use a decentralized algorithm. We show that by using carrierdifferential GPS, excellent performance is still achiev-

*In GPS terms, this can be either the single difference or double difference able using this decentralized approach. What we present in this work is the most general, most scalable, and most conservative realization of a formation state estimation algorithm.

\section{ESTIMATION}

Estimation is performed in real-time. There is no smoothing, or any "scouting passes" (as in the adaptive work by Sarkar et al. in Ref. [13]). The estimation is performed by filtering measurements from the space-capable, CDGPS receivers. There are two filters, an absolute state filter and a relative state filter. The focus of this work was mainly on the relative navigation performance and less on the absolute navigation performance. For absolute state estimation, a standard iterative least-square method is implemented using the code phase and Doppler measurements. The algorithm can be found in standard GPS texts. ${ }^{14}$ In general, the absolute position error is less than $\sim 20 \mathrm{~m}$ and the velocity error less than $\sim 0.2 \mathrm{~m} / \mathrm{s}$. It was found that the coupling between the relative and absolute solution is very weak, and absolute errors of even hundreds of meters have no observable impact on the relative solution.

An Adaptive Extended Kalman Filter has been designed for formation flying spacecraft. At each time-step new measurements are made available from two GPS receivers (the local slave receiver, and the master receiver). The single difference was taken on the code phase $(\rho)$, carrier phase $(\phi)$, and Doppler $(\dot{\phi})$ measurements for all commonly observed NAVSTAR signals. The number of measurements available at any time-step is different, making the system time-variant. Figure 2 shows the number of NAVSTAR satellites used over the time of a sample experiment; there is one measurement for each satellite. Since each carrier measurement has an associated bias, included in the state vector, this requires reformulation of every matrix in the filter at every time-step.

The state, $\mathbf{x}$, is defined at time-step $k$ as:

$$
\mathbf{x}_{k}=\left[\begin{array}{c}
\Delta \mathbf{r}_{i j} \\
\Delta b_{i j} \\
\Delta \dot{\mathbf{r}}_{i j} \\
\Delta \dot{b}_{i j} \\
\Delta \beta_{1} \\
\vdots \\
\Delta \beta_{n}
\end{array}\right]
$$




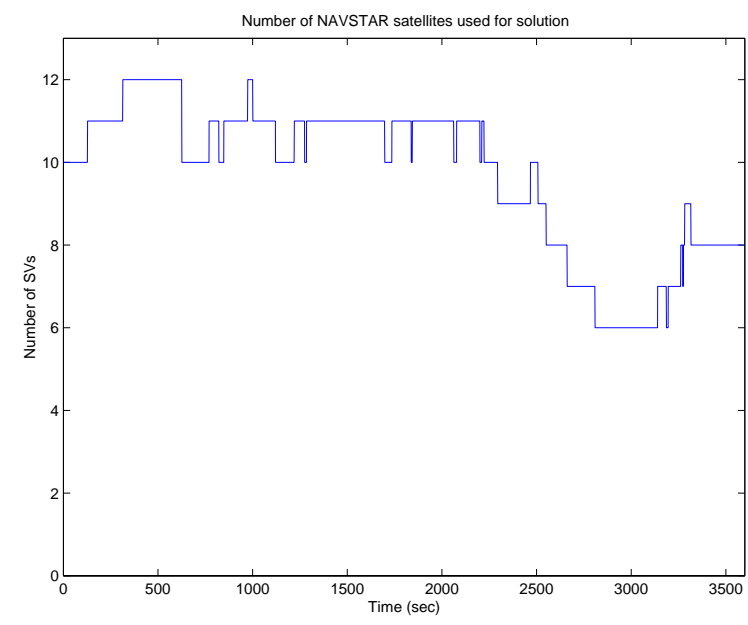

Fig. 2: Typical number of NAVSTAR satellites visible. Each change in number requires a change in the state and measurement vectors.

where $\Delta \mathbf{r}_{i j}$ and $\Delta \dot{\mathbf{r}}_{i j}$ are the relative position and velocity vectors between the slave $(j)$ and the master vehicle $(i), \Delta b_{i j}$ and $\Delta \dot{b}_{i j}$ are the differential receiver clock offset and clock drift from GPS system time, and $\Delta \beta$ are the differential carrier phase biases on each single difference measurement, where there are $n$ such measurements at a given time-step.

The measurements between GPS satellite $m$ and the $i^{t h}$ user are defined:

$$
\begin{aligned}
\rho_{i}^{m} & =\left|\Delta \mathbf{r}_{i}^{m}\right|+b_{i}+B^{m}+I_{i}^{m}+\nu_{\rho} \\
\phi_{i}^{m} & =\left|\Delta \mathbf{r}_{i}^{m}\right|+b_{i}+B^{m}-I_{i}^{m}+\beta_{i}^{m}+\nu_{\phi} \\
\dot{\phi}_{i}^{m} & =\left(\Delta \dot{\mathbf{r}}_{i}^{m}\right) \cdot \frac{\Delta \mathbf{r}_{i}^{m}}{\left|\Delta \mathbf{r}_{i}^{m}\right|}+\dot{b}_{i}+\dot{B}^{m}+\nu_{\dot{\phi}}
\end{aligned}
$$

where

$$
\begin{aligned}
b_{i}= & \text { clock offset for user } i \\
B^{m}= & \text { clock offset for GPS satellite } m \\
I_{i}^{m}= & \text { Ionospheric delay } \\
\beta_{i}^{m}= & \text { Carrier Phase bias between } i \text { and } m \\
\nu= & \text { other noises (including receiver front end, } \\
& \text { multipath, and unmodeled environment) }
\end{aligned}
$$

The term $\Delta \mathbf{r}_{i}^{m}$ is $\mathbf{r}^{m}\left(t^{s n d}\right)-\mathbf{r}_{i}\left(t_{r c v}\right)$, where $\mathbf{r}^{m}\left(t^{s n d}\right)$ is the GPS satellite state at the time the signal was sent, and $\mathbf{r}_{i}\left(t_{r c v}\right)$ is the receiver state at the time the signal was received.

A few important points should be made about the measurements. All work is done in the WGS-84 Earth Centered-Earth Fixed Cartesian coordinate frame. An Earth-Fixed frame changes with time and so all states are expressed in the frame at the time the master measurement was received. Special care is taken to express this GPS satellite state at transmission time in the master-receiver time ECEF frame.

The carrier phase measurement has an inherent bias, the integer cycle ambiguity. Since all carrier waves are identical, there is an unknown number of integer waves between any two points. Many techniques and methods exist for determining this integer number. ${ }^{15}$ For this work, however, the carrier phase bias was treated as a real number, and it includes the line biases as well. These biases were estimated as states in the relative filter, and there was no other filter or routine implemented to determine the carrier phase biases, such as we have done in previous work. ${ }^{8,9}$ In general, the biases were resolved within five minutes after initialization, and once the filter had converged, new biases were determined very rapidly.

The measurements are taken on each receiver, and then collected and aligned so that single differences were taken between measurements from the same GPS satellite. Double-differences and singledifferences were tried and both techniques demonstrated similar performance. Therefore single differences are used because they are easier to implement. The measurement vector used at time-step $k$ is

$$
y_{k}=h_{k}\left(\mathbf{x}_{k}\right)=\left[\begin{array}{c}
\Delta \phi_{i j} \\
\Delta \dot{\phi}_{i j}
\end{array}\right]
$$

The measurement vector $\Delta \phi_{i j}$ is the set of single differences between the master vehicle and slave vehicle,

$$
\Delta \phi_{i j}=\phi_{j}-\phi_{i}\left\{\begin{array}{l}
\forall j=1, n \\
i=\text { master }
\end{array}\right.
$$

There are $n=N-1$ single-difference sets, where $N$ is the number of vehicles in the formation. The length of the measurement vector depends on the number of GPS satellites in view, and can vary from four to twelve. To estimate the measurements, the nonlinear function, $\hat{h}_{k}$, is defined using

$$
\begin{aligned}
\Delta \hat{\phi}_{i j}^{m}= & \left|\Delta \hat{\mathbf{r}}_{j}^{m}\right|-\left|\Delta \hat{\mathbf{r}}_{i}^{m}\right|+\Delta \hat{b}_{i j}-\Delta I_{i j}^{m}+\Delta \beta_{i j}^{m} \\
\Delta \dot{\hat{\phi}}_{i j}^{m}= & \left(\Delta \dot{\hat{\mathbf{r}}}_{j}^{m}\right) \cdot \frac{\Delta \hat{\mathbf{r}}_{j}^{m}}{\left|\Delta \hat{\mathbf{r}}_{j}^{m}\right|}-\left(\Delta \dot{\hat{\mathbf{r}}}_{i}^{m}\right) \cdot \frac{\Delta \hat{\mathbf{r}}_{i}^{m}}{\left|\Delta \hat{\mathbf{r}}_{i}^{m}\right|} \\
& +\Delta \dot{\hat{b}}_{i j}
\end{aligned}
$$


where $\hat{\mathbf{r}}_{i}$ is the absolute position estimate for slave vehicle $i$, and is determined by $\hat{\mathbf{r}}_{1}+\Delta \hat{\mathbf{r}}_{i}$. The GPS satellite states, $\mathbf{r}^{m}$ and $\dot{\mathbf{r}}^{m}$, are the position and velocity of the GPS satellite at the time it transmitted the signal, and are determined from their broadcast ephemeris data. It is found through an iterative process using the time the signal was received and the distance the signal traveled. ${ }^{7}$

Given the state and measurements, the state estimate, $\hat{\mathbf{x}}_{k}$ is updated:

$$
\begin{aligned}
\hat{\mathbf{x}}_{k}^{+}= & \hat{\mathbf{x}}_{k}^{-}+K_{k}\left(y_{k}-\hat{h}_{k}\left(\hat{\mathbf{x}}_{k}^{-}\right)\right) \\
P_{k}^{+}= & \left(I-K_{k} H_{k}\right) P_{k}^{-}\left(I-K_{k} H_{k}\right)^{T} \\
& +K_{k} R_{k} K_{k}^{T}
\end{aligned}
$$

where the $(\cdot)^{+}$and $(\cdot)^{-}$indicate a posteriori and $a$ priori. $P_{k}$ is the covariance of the state estimate. $R_{k}$ is the sensor noise covariance. The matrix $H$ is the first order Taylor expansion of $\hat{h}$ evaluated at $\hat{\mathbf{x}}_{k}^{-}$. $K_{k}$ is the Kalman gain,

$$
K_{k}=P_{k}^{-} H_{k}^{T}\left(H_{k} P_{k}^{-} H_{k}^{T}+R_{k}\right)^{-1}
$$

The state estimate and covariance is then propagated between time-steps.

$$
\begin{aligned}
\hat{\mathbf{x}}_{k+1}^{-} & =f\left(\hat{\mathbf{x}}_{k}^{+}, \Delta t\right) \\
P_{k+1}^{-} & =\Phi_{k} P_{k}^{+} \Phi_{k}^{T}+Q_{k}
\end{aligned}
$$

The nonlinear function, $f\left(\hat{\mathbf{x}}_{k}^{+}, \Delta t\right)$ is the continuous differential equation for the state over the time interval $\Delta t$. It is evaluated using a fourth order RungeKutta numerical integration. $\Phi_{k}$ is the discretized first order Taylor expansion of $f(\cdot)$, and is recalculated every time-step. $Q_{k}$ is the discrete covariance matrix for the process noise.

The differential state propagation uses only a simple Keplerian orbit model. There are no higher order gravity terms, no drag model, nor any other disturbance model. The work presented here used $\Delta t$ of 1 second. This very simple orbital model was sufficient for the differential motion over this short propagation period.

\section{Initialization}

There are four input parameters for any Kalman filter. They are the initial state estimate $\hat{\mathbf{x}}_{0}^{-}$, the initial covariance, $P_{0}^{-}$, the sensor noise covariance $R_{k}$, and the process noise covariance $Q_{k}$.
The initial state estimate is determined by taking the least-square solution of the differential code phase measurements $(\Delta \rho)$. For close formations (vehicles less than $1 \mathrm{~km}$ apart), this method gives an initial estimate with an accuracy of $2-4 \mathrm{~m}$. However, as the separation between vehicles grows, the error in the least squares solution grows as well (because the common line-of-sight assumption begins to break down). To compensate, Park's algorithm ${ }^{11}$ for correcting measurements is used on the code phase measurements. Using this technique, separations of up to 100 's of kilometers can still be initially determined to within a few meters. The correction is applied to the measurements,

$$
\Delta \rho_{i}^{m}=\Delta \rho_{i}^{m}+\epsilon_{\Delta \rho}^{m} \text { and } \Delta \dot{\phi}_{i}^{m}=\Delta \phi_{i}^{m}+\epsilon_{\Delta \dot{\phi}}^{m}
$$

with

$$
\begin{aligned}
\epsilon_{\Delta \rho}^{m} & =\left|\hat{\mathbf{r}}_{1}-\hat{\mathbf{r}}^{m}\right|\left(1-\operatorname{los}_{1}^{m} \cdot \operatorname{los}_{2}^{m}\right) \\
\epsilon_{\Delta \dot{\phi}}^{m} & =\left(\operatorname{los}_{2}^{m}-\operatorname{los}{ }_{1}^{m}\right) \cdot\left(\dot{\hat{\mathbf{r}}}_{2}-\dot{\hat{\mathbf{r}}}^{m}\right)
\end{aligned}
$$

The term $l s_{i}$ refers to the unit vector along the line-of-sight between the receiver indicated in the subscript and the $m^{t h}$ GPS satellite, in the ECEF frame. The subscripts 1 and 2 refer to the master and slave vehicle respectively. With the corrected measurements, the initial state estimate is found

$$
\begin{aligned}
G & =\left[\begin{array}{cc}
-\operatorname{los}_{1}^{1} & 1 \\
\vdots & \vdots \\
-\operatorname{los}_{1}^{n} & 1
\end{array}\right] \\
G^{\dagger} & =\left(G^{T} G\right)^{-1} G^{T} \\
\hat{\mathbf{x}}_{0}^{-} & =\left[\begin{array}{cc}
G^{\dagger} & 0 \\
0 & G^{\dagger}
\end{array}\right]\left[\begin{array}{c}
\Delta \boldsymbol{\rho} \\
\Delta \dot{\boldsymbol{\phi}}
\end{array}\right]
\end{aligned}
$$

Since the initial estimate is determined by the code phase estimate, the initial covariance is set to reflect this. It is determined upon initialization as follows

$$
\begin{aligned}
P_{\Delta r} & =G^{\dagger} R_{\Delta \rho}\left(G^{\dagger}\right)^{T} \\
P_{\Delta \dot{r}} & =G^{\dagger} R_{\Delta \dot{\phi}}\left(G^{\dagger}\right)^{T} \\
P_{0}^{-} & =\left[\begin{array}{cc}
\operatorname{diag}\left(P_{\Delta r}\right) & 0 \\
0 & \operatorname{diag}\left(P_{\Delta \dot{r}}\right)
\end{array}\right]
\end{aligned}
$$

Only the diagonal of the sub-matrices $P_{\Delta r}$ and $P_{\Delta \dot{r}}$ are used to insure that $P_{0}$ is positive definite. $P_{\Delta r}$ 
and $R_{\Delta \rho}$ refer to the sub-block within those matrices (on the diagonal) corresponding to the quantity in the subscript.

As the satellites move along the orbit, new GPS satellites will come into view, and new carrier biases will need to be added to the state. The initial estimate of the biases is set by

$$
\Delta \hat{\beta}_{i j}^{m}=\Delta \phi_{i j}^{m}-\left(\left|\hat{\mathbf{r}}^{m}-\hat{\mathbf{r}}_{i}\right|-\left|\hat{\mathbf{r}}^{m}-\hat{\mathbf{r}}_{j}\right|+\Delta \hat{b}_{i j}\right)(25)
$$

and the initial covariance for that bias estimate is

$$
P_{\beta}=G P_{\Delta r}^{-} G^{T}+\sigma_{\Delta \phi}^{2}
$$

where $\sigma_{\Delta \phi}^{2}$ is the covariance of the expected noise on the $\Delta \phi$ measurement.

\section{Adaptation}

To determine the noise covariance matrices, $R$ and $Q$, adaptive filter routines have been implemented. The values for these covariances may not be well modeled, especially in the case of $Q$. These parameters greatly influence the performance of the filter, and in some cases, if $Q$ is set too low, the filter will diverge. Therefore, it is very important to correctly identify them. The adaptive routines used are modified from algorithms summarized by Maybeck. ${ }^{16}$

The $R$ adaptation scheme works very well for all experimental scenarios. For this work, the real values of $R_{\Delta \phi}$ were $\sim(6 \mathrm{~mm})^{2}$ and $R_{\Delta \dot{\phi}}$ were $\sim(5$ $\mathrm{cm} / \mathrm{sec})^{2}$. The initial guess for $\hat{R}_{\Delta \phi}$ was set at $(10 \mathrm{~m})^{2}$ and $\hat{R}_{\Delta \dot{\phi}}$ was set at $(10 \mathrm{~m} / \mathrm{s})^{2}$. Even with these extreme initial guesses, the filter was able to correctly estimate $R$ within 100 seconds.

The process noise proved more difficult. The differential receiver clock noise is several orders of magnitude larger than the differential accelerations acting on the vehicle dynamics. The error introduced by the clock noise in effect "washed out" any noise/uncertainty in the vehicle dynamics. However, by holding the motion noise terms of $\hat{Q}$ constant, at least the clock noise $\left(Q_{\text {clock }}\right)$ could be identified through adaptation.

The $Q$ matrix is also adapted during active control. If thrusters are commanded to fire, that command is also sent to the filter. This thruster input is included in the state propagation. At this time, the $Q$ matrix is also increased to account for the fact that uncertainty in thruster behavior will often be far greater than those in the naturally occurring dif- ferential orbital process noises. The filter performed well while running the $R$ and $Q_{\text {clock }}$ adaptation algorithms simultaneously. The adaptive filter not only allowed identification of the filter parameters, but also improved the general filter performance by feeding back the residuals into the covariance update and propagation. A more detailed presentation of the adaptive filtering algorithms is presented in Ref. [17].

\section{DEMONSTRATION}

The GPS receiver used for this work is a modified receiver based on the GP2015 and GP2021 chipset by Zarlink (formerly Mitel/Plessey). The chipset has space heritage. ${ }^{18}$ The receiver design has previous terrestrial formation application heritage as well. ${ }^{19}$ It has variable gain Phase Lock Loop (PLL) carrier tracking loops. Special modifications were also made to allow operation at orbital altitudes and velocities. A simple orbit propagator insures the ability to acquire navigation fix even in orbit, which is necessary because of the larger Doppler search space. ${ }^{20}$ Relative navigation also requires simultaneous measurements, and so the receiver clock actively steers to GPS time. These modifications make this receiver an ideal orbital relative navigation sensor.

This receiver has already been extensively tested on the ground. Ground tests provide a performance baseline, especially in determining signal noise levels. With the multipath environment and troposphere, the ground test signal to noise ratios should be conservative bounds for what will be experienced on orbit.

The NASA Goddard Space Flight Center has a state-of-the-art Formation Flying Test-Bed. At its heart is the Spirent STR Series Multichannel Satellite Navigation Simulator. ${ }^{21}$ It uses a Compaq DS10 workstation to simulate the motion of the NAVSTAR constellation and up to 4 independent vehicles. The workstation sends these states to two STR4760 $\mathrm{RF}$ signal generators. These signal generators create the GPS signals that would be observed by the four user receivers (with two RF outputs per generator). Figure 3 shows a simple block diagram of the experimental set-up.

A variety of scenarios have been run using this orbital simulator. For this paper, a series of different formations based on the same master orbit have been run. The orbit simulated has an altitude of 


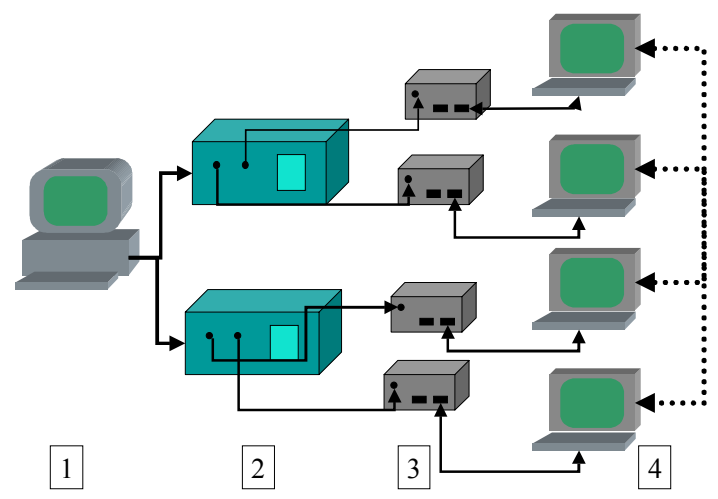

Fig. 3: This figure shows the GSFC FFTB setup. The features are: 1) a DS10 Workstation, which sends orbital states to 2) the STR4760 GPS signal generators, which sends actual RF signals, received by 3) the GPS receivers, which perform real-time absolute navigation and also collect measurements, which are saved on 4) data storage laptops

$\sim 450 \mathrm{~km}$, eccentricity of 0.005 , and $28.5^{\circ}$ inclination. The simulation used a tenth-order gravity model and an atmospheric drag model. For the measurements, a diverging ephemeris and clock model was used and a standard orbital ionospheric model, as specified in NATO Standard Agreement STANAG 4294 Issue 1 . There is no multipath noise in the simulation, but this is not expected to be large problem for microsatellites. All vehicle models are identical, with a surface area of $1 \mathrm{~m}^{2}$, drag coefficient of 2 , and 0.1 metric tonne (the smallest mass the simulator would model). The antenna is at the center of gravity, and the vehicle maintained a nadir earth-pointing attitude. A standard hemispherical antenna gain pattern was used.

A MATLAB simulation environment was also developed. It allows using either or both of the true motion files saved by the FFTB workstation and the GPS measurement files collected on the laptops. Among other functions, white process noise and measurement noise can be used in place of the more realistic (and less ideal) colored noises which exist in the hardware data. This makes it possible to compare the effects of these non-ideal conditions on the estimation performance.

\section{RESULTS}

This section presents the results from real-time hardware in the loop experiments using the GSFC FFTB facility. A series of experiments with different forma-
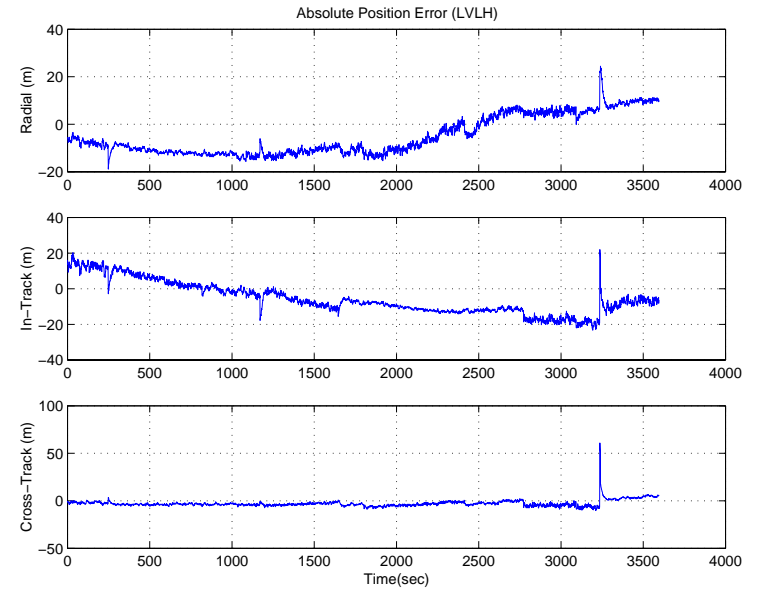

Fig. 4: Typical absolute position error over time during one of the real-time hardware-in-the-loop experiments. The absolute state estimates are using only weighted least square estimation.

tions and different focuses were performed. For all experiments, data storage of the raw measurements began after all four of the receivers achieved navigation fixes. The receivers performed "warm" starts, where they were provided almanac information for the NAVSTAR constellation, a rough current position guess (often wrong by several hundred kilometers) and a rough current time guess (off by up to fifteen seconds). The receivers reliably and repeatedly locked on in all tests conducted with the latest software (accomplished approximately 75 times). A navigation fix was usually attained, and active tracking begun, in less than two minutes after the simulation began. Figures 4 and 5 show typical absolute position and velocity errors over time during a sample experiment. Absolute estimation was performed using least-squares. Little effort was made to improve absolute state estimation in order to demonstrate the robustness of the relative navigation even with sub-optimal absolute estimates.

To establish baseline performance, tests were run using a basic formation. The initial orbital elements given in Table 1 create an in-plane formation of three vehicles moving in an ellipse around a fourth, central vehicle, with a radius on the order of $1 \mathrm{~km}$. Figure 6 shows the in-plane, LVLH frame projection of the motion of the vehicles for a portion of this experiment (only a short period was shown to maintain clarity). The three slave vehicles each compute a differential solution with respect to the master vehicle simultaneously and independently. 
Table 1: Initial Orbital Parameters for $1 \mathrm{~km}$ In-Plane Elliptical Formation

\begin{tabular}{|c|c|c|c|c|c|c|}
\hline Veh. & $a(m)$ & $\mathrm{e}$ & $i\left(^{\circ}\right)$ & $\Omega\left(^{\circ}\right)$ & $\omega\left(^{\circ}\right)$ & $M\left(^{\circ}\right)$ \\
\hline 1 & $\overline{7017995.7}$ & 0.005000 & $\overline{28.5}$ & 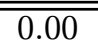 & $\overline{-88.76}$ & $\overline{135.00}$ \\
\hline 2 & 7017988.6 & 0.005103 & 28.5 & -1.13 & -88.76 & 136.13 \\
\hline 3 & 7018005.0 & 0.004862 & 28.5 & -0.43 & -88.76 & 135.42 \\
\hline 4 & 7017992.8 & 0.005038 & 28.5 & 1.59 & -88.76 & 133.44 \\
\hline
\end{tabular}
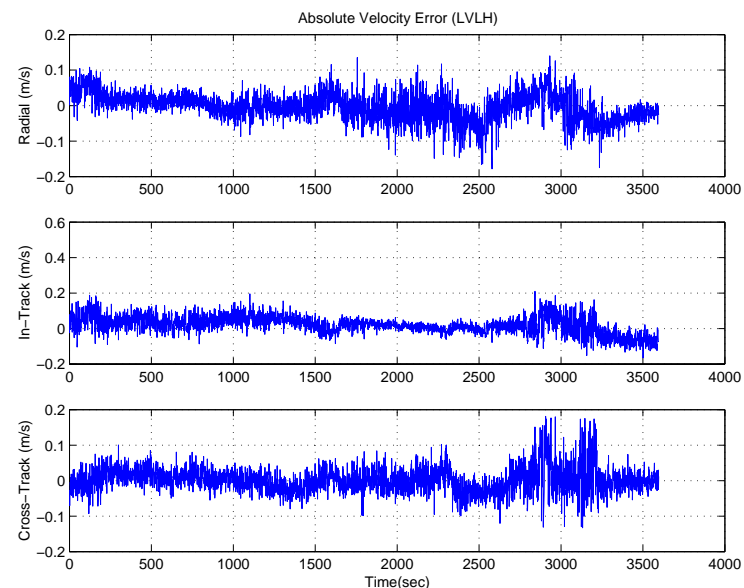

Fig. 5: Typical absolute velocity error over time during one of the real-time hardware-in-the-loop experiments. The absolute state estimates are using only weighted least square estimation.

For four vehicles, there are three independent differential solutions. Our primary interest is in the relative state error, where the error is defined as $\tilde{x}=\hat{x}-x$. Figure 7 shows the position error and figure 8 shows the velocity error for the three relative solutions. The plots correspond to the projections of the error into the Radial, In-Track, and Cross-Track directions. During the first two hundred seconds, there are large errors (on the order of $2-5 \mathrm{~m}$, sometimes up to $10-15 \mathrm{~m}$ ). This initial position error is due to the initial carrier phase bias estimate errors. Over time, using the change in lines-of-sight, the biases become observable, and the position solution converges. The velocity solution is coupled with the position, and also improves as the position is determined.

To quantify performance, the standard deviation and mean of the error after filter convergence is computed over time. Table 2 gives the root-mean-square of these means and standard deviations of the errors across the fleet's three solutions. The values are given for the Radial (R), In-Track (I), and Cross-

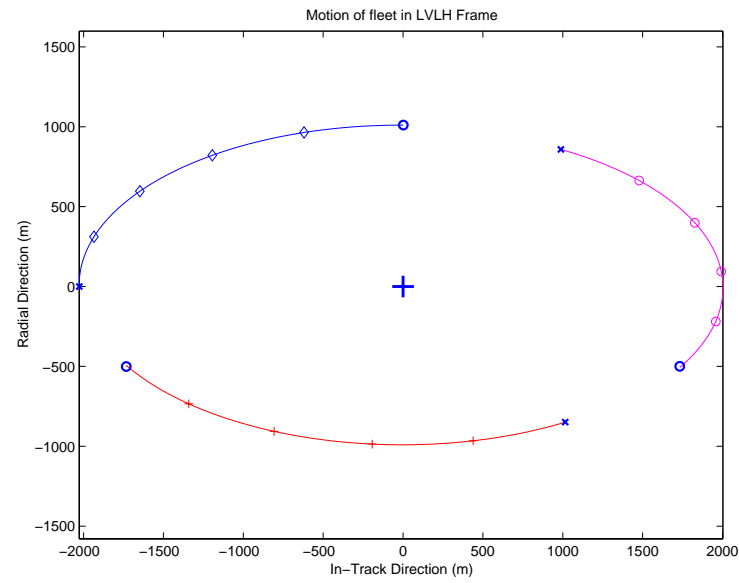

Fig. 6: The initial in-plane elliptical motion of three slave vehicles (starting at the "o" positions) around a master node (marked by "+")

Table 2: Relative State Estimation Results: $1 \mathrm{~km}$ In-Plane Elliptic Formation

\begin{tabular}{|l|c||cc|}
\hline \multicolumn{2}{|c|}{ Rel. State Error } & Mean $(\mu)$ & St. Dev. $(\sigma)$ \\
\hline \hline \multirow{3}{*}{ Position $(\mathrm{cm})$} & R & 0.91 & 0.59 \\
& I & 1.71 & 0.36 \\
& C & 0.42 & 0.23 \\
\hline \multirow{3}{*}{ Velocity $(\mathrm{cm} / \mathrm{s})$} & R & 0.007 & 0.023 \\
& I & 0.003 & 0.032 \\
& C & 0.006 & 0.021 \\
\hline
\end{tabular}

Track (C) directions. The position error has a mean of $0.4-1.7 \mathrm{~cm}$, and a standard deviation of $0.23-0.59 \mathrm{~cm}$. The fact that the mean is larger than the standard deviation is probably a result of the carrier phase bias estimation process. The velocity error has a mean of $0.03-0.07 \mathrm{~mm} / \mathrm{s}$ and a standard deviation of $\sim 0.3 \mathrm{~mm} / \mathrm{s}$. The velocity mean error is extremely low, which is very good, because any active control algorithm is most sensitive to the velocity error mean. To the author's knowledge, these are the lowest position errors, and among the lowest velocity errors, ever reported for orbital relative navigation. 

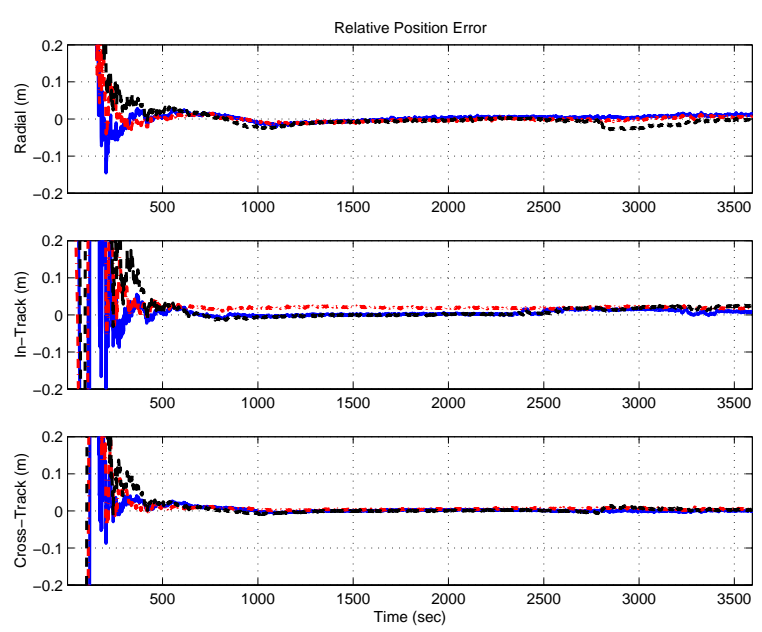

Fig. 7: Position Error for three independent simultaneous solutions for $1 \mathrm{~km}$ in-plane ellipse during hardware-in-the-loop experiments

Table 3: Relative State Estimation Results: $10 \mathrm{~km}$ In-Plane Elliptic Formation

\begin{tabular}{|l|c||cc|}
\hline \multicolumn{2}{|c|}{ Rel. State Error } & Mean $(\mu)$ & St. Dev. $(\sigma)$ \\
\hline \hline \multirow{3}{*}{ Position $(\mathrm{cm})$} & $\mathrm{R}$ & 3.87 & 1.19 \\
& $\mathrm{I}$ & 2.58 & 1.18 \\
& $\mathrm{C}$ & 1.98 & 0.63 \\
\hline \multirow{3}{*}{ Velocity $(\mathrm{cm} / \mathrm{s})$} & $\mathrm{R}$ & 0.022 & 0.13 \\
& $\mathrm{I}$ & 0.006 & 0.21 \\
& $\mathrm{C}$ & 0.014 & 0.16 \\
\hline
\end{tabular}

The separation of the vehicles within the formation impacts the performance of the filter. This is not only because of increased nonlinearity, but more importantly because the differential process noise grows larger as the vehicles are farther apart. Table 3 shows the RMS mean and standard deviations from a $10 \mathrm{~km}$ in-plane elliptical formation. Here position errors are on the order of $2-4 \mathrm{~cm}$, and velocity errors on the order of $1-2 \mathrm{~mm} / \mathrm{s}$. Comparing Tables 3 and 2 reveals a material degradation in the performance of the estimator.

Also of interest is the ability to estimate the state during active formation maneuvers, i.e., using thrusters. Sample control inputs were performed during the simulation using a script of previously generated control commands. An experiment was performed using a 3-vehicle formation, where the formation was changed from an in-track formation to an in-plane elliptical formation (an In-track formation has the vehicles moving in the same orbit one after the other). The commands are fed forward
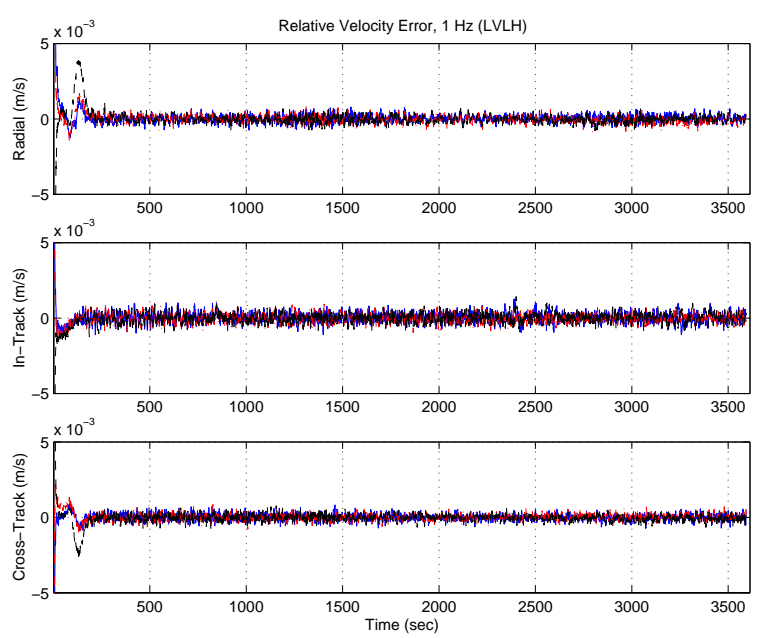

Fig. 8: Velocity Error for three independent simultaneous solutions for $1 \mathrm{~km}$ in-plane ellipse during hardware-in-the-loop experiments

to the Kalman filter. However, to demonstrate robustness, the value fed to the filter was changed $5 \%$ to simulate uncertainty in actual thruster behavior. Figure 9 shows the relative velocity of one of the vehicles. The 10 second thruster firing of $0.1 \mathrm{~m} / \mathrm{s}^{2}$ can clearly be seen by the change in the radial velocity. Figure 10 shows the velocity error, and figure 11 shows the position error for the two relative states during the experiment. Both slave vehicles fire their thrusters at the same time (in opposite radial directions). During the actual thruster firing, the velocity estimate jumps. This is partly due to the $5 \%$ simulated error, and also because of the increase in the $Q$ matrix during active control. The important point of these results is that the estimate returns almost immediately to the previous accuracy level directly after the thruster firing. The position estimate maintains accuracy throughout the thruster firing.

\section{CONCLUSION}

We have experimentally demonstrated real-time decentralized precision relative navigation using Carrier Differential GPS. Using a modified low-cost commercially available GPS receiver, orbital absolute navigation has been repeatably demonstrated. Precise code phase, carrier phase, and Doppler measurements have been collected and analyzed. An adaptive extended Kalman filter has been developed to take those measurements and provide relative state estimation for vehicles within a variety of forma- 

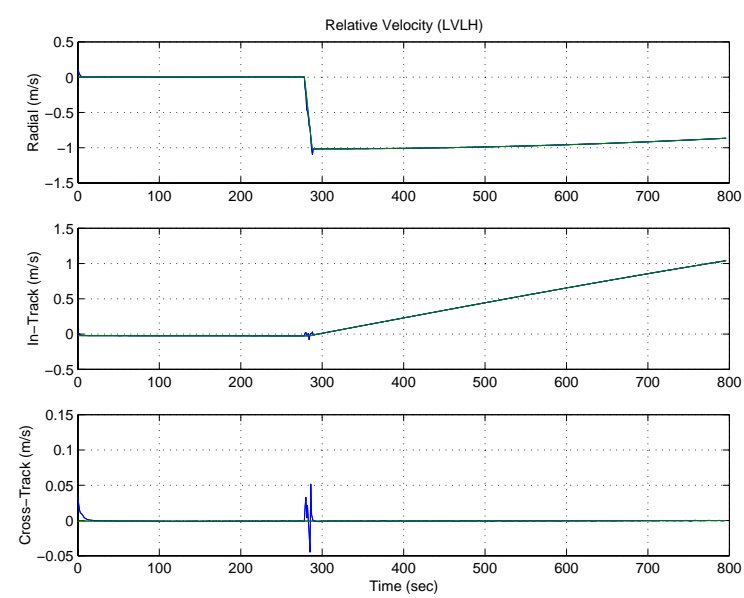

Fig. 9: Shows the thrust during a formation maneuver by one of the slave vehicles; the commanded thrust was $\Delta V \approx-1 \mathrm{~m} / \mathrm{s}$ over a 10 second period in the radial direction.
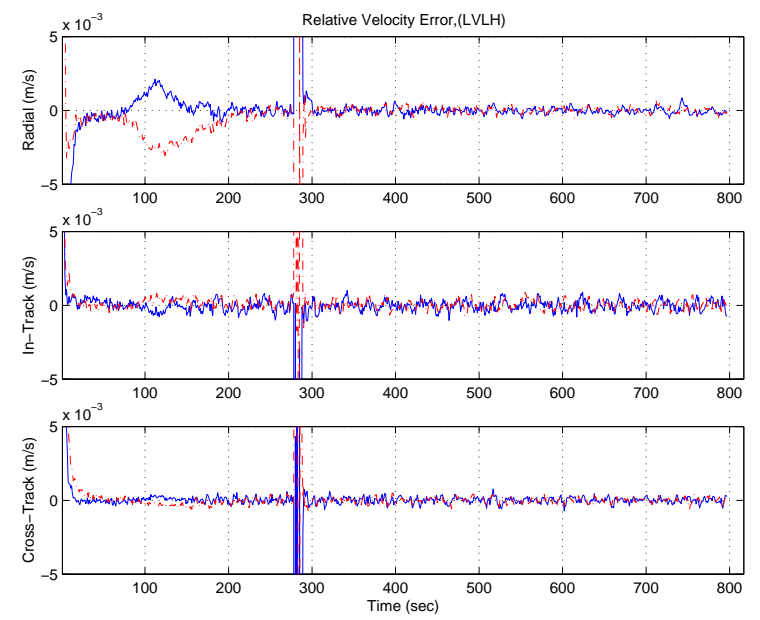

Fig. 10: Velocity Error for two differential solutions during a commanded maneuver by both vehicles

tions. Even during maneuvers with poorly modeled accelerations, state estimation with unprecedented accuracy can be achieved. For formations with kilometer separations between vehicles, accuracies of $1 \mathrm{~cm}$ position error and $0.5 \mathrm{~mm} / \mathrm{s}$ velocity error has been experimentally demonstrated.

\section{ACKNOWLEDGMENTS}

We gratefully acknowledge J. Simpson, R. Burns, J. Leitner, and F. Bauer at NASA Goddard Space Flight Center for their help and support in developing and testing this receiver using the FFTB. This work was funded through NASA grant NAG5-10719. This work in many ways is an extension of work done
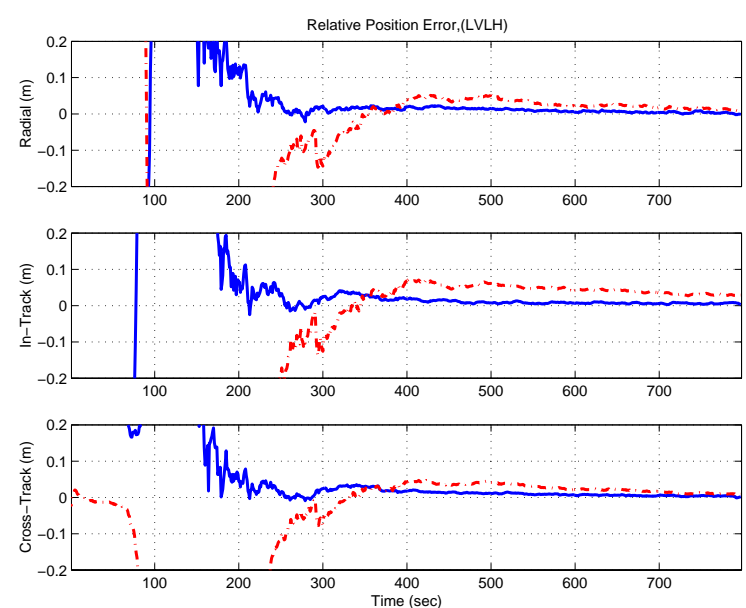

Fig. 11: Position Error for two differential solutions during a commanded maneuver by both vehicles

by Chan Woo Park, and we also acknowledge his contribution to this work.

\section{REFERENCES}

[1] F.H.Bauer, K. Hartman, J.P. How, J. Bristow, D. Weidow, F. Busse, "Enabling Spacecraft Formation Flying through Spaceborne GPS and Enhanced Automation Technologies," Proc. of Institute of Navigation, July 1999

[2] J. Leitner, F. Bauer, D. Folta, R. Carpenter, M. Moreau, and J.P. How, "Formation Flight in Space," GPS World, Feb. 2002, pp. 22-31.

[3] J. How and M. Tillerson, "Analysis of the Impact of Sensor Noise on Formation Flying Control," Proceedings of the American Control Conference, pp. 3986-3991, 2001.

[4] M. Tillerson, Coordination and Control of Multiple Spacecraft using Convex Optimization Techniques, S.M. Thesis, Department of Aeronautics and Astronautics, MIT, June 2002.

[5] I. Kawano, M. Mokuno, T. Kasai, T. Suzuki, "First Autonomous Rendezvous Using Relative GPS Navigation by ETS-VII," Journal of the Institute of Navigation, Vol. 48, No. 1, Spring 2001.

[6] J. R. Carpenter, E. Schiesser, "Semi major Axis Knowledge and GPS Orbit Determination," Journal of the Institute of Navigation, Vol. 48, No. 1, Spring 2001.

[7] T. Ebinuma,R. Bishop, E. Lightsey, "Hardware-inthe-Loop GPS Test Facility for Spacecraft Autonomous Rendezvous," Proceedings of ION GPS, Salt Lake City, UT, Sep. 2001. 
[8] F.D. Busse, G. Inalhan, J.P. How, "Project Orion: Carrier Phase Differential GPS for Formation Flying," Proc. of AAS Guidance and Control Conference, Breckenridge, CO, Feb 2000.

[9] F.D. Busse, J.P. How, J. Simpson, J. Leitner, "Orion-Emerald: Carrier Differential GPS for LEO Formation Flying," Proc. of IEEE Aerospace Conference, Big Sky, MT, Mar 2001.

[10] J.R. Carpenter, "Decentralized Control of Satellite Formations," International Journal of Robust and Nonlinear Control, 2002, 12:141-161.

[11] C.W. Park, Precise Relative Navigation Using Augmented CDGPS, Ph.D. Dissertation, Stanford University, Dept. of Mechanical Engineering, June 2001.

[12] P. Binning, I. Galysh, "Satellite to Satellite Relative Navigation Using GPS Pseudoranges," Proc. of ION National Technical Meeting, Santa Monica, CA, Jan. 1997.

[13] A. Sarkar, M. Ananthasayanam, S. Vathsal, "Sensitivity of Initial State Error Covariance Matrix in a Practical Adaptive EKF," AIAA Atmospheric Flight Mechanics Conference, Montreal, Canada, Aug. 2001, AIAA-2001-4202.

[14] P. Axelrad, R.G. Brown, "GPS Navigation Algorithms," Global Positioning System: Theory and Applications, Vol. I, ed. B.W. Parkinson, J.J. Spilker Jr., AIAA, 1996.

[15] S. Han, C. Rizos, "Comparing GPS Ambiguity Resolution Techniques," GPS World, Oct. 1997.

[16] P. Maybeck, Stochastic Models, Estimation, and Control, Volume 2, Academic Press, New York, 1982.

[17] F.D. Busse, J.P. How, J. Simpson, R. Burns, "Adaptive EKF for Precise Real-Time Orbital Relative Navigation," Institute of Navigation GPS Conference, Portland, OR, Sep. 2002.

[18] M.J. Unwin, P.L. Palmer, Y. Hashida, C.I. Underwood, "The SNAP-1 and Tsinghua-1 GPS Formation Flying Experiment," Proc. of Institute of Navigation, Salt Lake City, UT, Sep 2000.

[19] E. Olsen, GPS Sensing for Formation Flying Vehicles, Ph.D. Thesis, Stanford University, Dept. of Aeronautical and Astronautical Engineering, Dec 1999.

[20] E. G. Lightsey, Development and Flight Demonstration of a GPS Receiver for Space, Ph.D. Thesis, Department of Aeronautics and Astronautics," Stanford University, Feb., 1997.

[21] STR Series Multichannel Satellite Navigation Simulator Reference Manual, May 2001. 